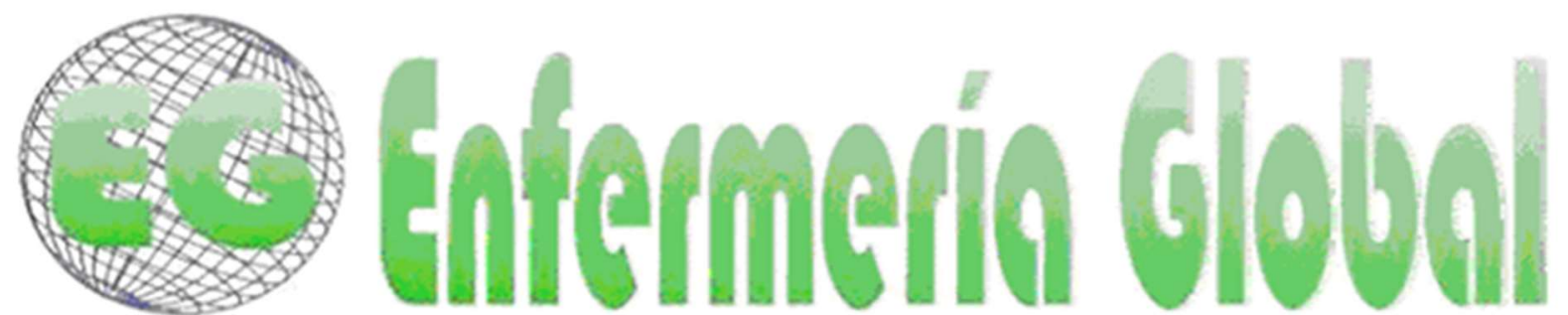

\title{
ORIGINALES
}

\section{La concentración de fluoruro en las aguas consumidas en la Región de Murcia no es suficiente para prevenir la caries dental}

Fluoride concentrations in water consumed in the Region of Murcia are not sufficient to prevent dental caries

\author{
Amparo Pérez Silva ${ }^{1}$ \\ Jaime Aparecido Cury ${ }^{2}$ \\ Clara Serna Muñoz ${ }^{1}$ \\ Inmaculada Cabello Malagón ${ }^{1}$ \\ Yolanda Martinez Beneyto ${ }^{1}$ \\ Antonio José Ortiz Ruiz ${ }^{1}$
}

1 Departamento de Odontología Integrada Infantil. Facultad de Medicina. Universidad de Murcia. Instituto de Investigaciones Biosanitarias de la Región de Murcia (IMIB) Murcia, España. icabello@um.es

${ }^{2}$ Departamento de Bioquímica. Facultad de Odontología de Piracicaba, UNICAMP, Piracicaba, SP, Brasil.

\section{https://doi.org/10.6018/eglobal.432151}

Recibido: $12 / 06 / 2020$

Aceptado: 4/10/2020

\section{RESUMEN:}

Introducción: La caries sigue siendo la enfermedad crónica más frecuente en el niño y es considerada un problema de salud pública. El flúor es la principal medida protectora contra la caries dental y el agua de consumo es la principal fuente de ingesta de flúor.

Objetivo: El objetivo de nuestro trabajo fue calcular la concentración de ión fluoruro en el agua de abastecimiento de 10 zonas y en 10 aguas embotelladas comercializadas en la Región de Murcia.

Material y Métodos: La concentración de fluoruro en las aguas se determinó con un electrodo ionselectivo para flúor previamente calibrado con patrones de fluoruro preparados con TISAB II.

Resultados: En el agua de abastecimiento, se detectaron concentraciones de fluoruro entre 0.09 y $0.18 \mathrm{mgF} / \mathrm{L}$ (ppm) en las aguas embotelladas la concentración de fluoruro varió desde 0.04 a 0.50 ppm. Conclusiones: El fluoruro está presente en aguas consumidas en la Región de Murcia pero en concentraciones que no alcanzan niveles preventivos para la caries dental. Es necesario prescribir suplementos de flúor en niños con alto riesgo de caries y para ello se deben contabilizar todas las fuentes externas de flúor, incluido el agua.

Palabras clave: Flúor, fluoruro, caries dental, agua mineral, agua de bebida.

\section{ABSTRACT:}

Introduction: Dental caries remains the most frequent chronic disease in childhood and is considered a public health problem. Fluoride has proven effectiveness in the prevention of caries and drinking water is the main source of fluoride intake. The objective of this study was to determine fluoride concentrations in tap water from 10 areas and in 10 bottled waters sold in the Region of Murcia. 
Materials and Methods: The concentration of fluoride in water was determined using a fluoride ionselective electrode with a direct technique previously calibrated with standard fluoride concentrations prepared with TISAB II.

Results: In tap water, fluoride concentrations from 0.09 to $0.18 \mathrm{mgF} / \mathrm{L}$ (ppm) were detected; in bottled waters the concentration of fluoride varied from 0.04 to $0.50 \mathrm{ppm}$.

Conclusion: Fluoride is present in the water consumed in the Region of Murcia but in concentrations that do not prevent dental caries. Fluoride supplements should be prescribed in children at high risk of caries, and for this, all external sources of fluoride intake, including water, must be accounted for.

Key words: Fluoride, dental caries, mineral water, tap water.

\section{INTRODUCCIÓN}

Para la Organización Mundial de la Salud (OMS) la caries dental sigue siendo la enfermedad crónica más frecuente en el niño en la mayoría de los países, teniendo un impacto negativo en la calidad de vida del niño y sus familias, y es considerada un problema de salud pública ${ }^{(1,2)}$.

El estudio Global Burden of Disease 2015 concluyó que en todo el mundo el índice de prevalencia estandarizado por edad de caries no tratadas en dentición temporal fue del 7.8\% (573 millones de niños) y la incidencia de nuevos casos de caries en dentición temporal fue de 126 millones de niños en $2015^{(3)}$. En España la última encuesta de salud oral mostró una prevalencia de caries en dentición temporal del $31,5 \%$ a los 5-6 años y en dentición permanente del $33,3 \%$ a los 12 años y del $43.2 \%$ a los 15 años ${ }^{(4)}$.

El agua es una de las principales fuentes de ingesta de flúor. En odontología, el contenido de flúor en el agua es relevante, pues su presencia puede tener consecuencias beneficiosas o perjudiciales para el paciente, dependiendo de su concentración ${ }^{(5)}$.

La fluoración de las aguas de consumo como medida preventiva, segura y eficiente, contra la caries se introdujo en el siglo pasado. Dentro de la Unión Europea, sólo el Reino Unido, Irlanda y España regularon la fluoración que, usualmente, se efectúa por adición de fluoruro sódico, ácido hexafluorosilícico o ácido fluorsilícico. Es un método reconocido en la prevención de la caries dental cuando se utiliza en concentraciones adecuadas para cada región, según la temperatura local ${ }^{(6,7)}$. Sin embargo, en el año 2014 el agua de la red pública del $95 \%$ de las ciudades españolas contenía menos de 0,7 ppm de flúor, ya que se abandonó la fluoración de las aguas de abastecimiento público debido al aumento del consumo de agua embotellada (8). En la comunidad autónoma de la Región de Murcia, en el año 2013, las aguas de Murcia dejaron de fluorarse.

La concentración de flúor en el agua, para que tenga un efecto protector contra la caries, debe de ser de 0,7 ppm según la American Academy of Pediatric Dentistry (AAPD) ${ }^{(9)}$, o estar entre 0,6 y 0,8 ppm según el Ministerio de Sanidad español (RD $1798 / 2010$ de 30 de diciembre) ${ }^{(10)}$. De hecho, a los niños que beban agua con menos de 0,6 ppm de flúor y que presenten alto riesgo de caries se les debe de prescribir suplementos orales de flúor ${ }^{(9)}$.

El agua mineral embotellada es la bebida que más se consume en España, siendo el cuarto productor en la Unión Europea y el quinto en consumo, a pesar de que los 
expertos aseguran que el agua del grifo es igual de buena, tiene los mismos minerales, y una huella ambiental muchísimo menor. En la región de Murcia, dependiendo de las mezclas de agua procedente del Segura, agua del Tajo y agua desalada del mar, el sabor varía sustancialmente, motivo por el cual el $50 \%$ de la población murciana consume agua embotellada, mineral o mineralizada. Murcia es una provincia donde hay pocas precipitaciones y se alcanzan altas temperaturas, con más de 300 días de sol al año. En el año de 1994, obtuvo el récord absoluto de temperatura máxima registrada en España en todo el siglo XX, con $47,2^{\circ} \mathrm{C}$. Estas razones son suficientes para que el consumo de agua del grifo o mineral sea bastante elevado ${ }^{(11)}$.

El objetivo de nuestro trabajo fue analizar la concentración de flúor en las aguas consumidas, embotelladas y de abastecimiento, de la región de Murcia.

\section{MATERIAL Y MÉTODOS}

\section{Muestras}

\section{Aguas embotelladas}

Se seleccionaron un total de 10 marcas de agua mineral y mineralizada frecuentemente encontradas en los comercios de la Región de Murcia (España). Todas las aguas estaban envasadas en botellas de plástico, salvo la Vichy Catalán que estaba envasada en botella de cristal, y fueron almacenadas a temperatura ambiente, resguardadas de la luz del sol y de la humedad hasta el momento del análisis. En una de las marcas las aguas procedían de dos fuentes naturales diferentes. La procedencia de las aguas embotelladas fue: Alicante, Badajoz, Cuenca, Gerona, Granada, Murcia, Pontevedra, Valencia. La composición que aparecía en sus etiquetas queda recogida en la Tabla 1.

Tabla 1: Composición en el etiquetado de aguas minerales y mineralizadas consumidas en la región de Murcia.

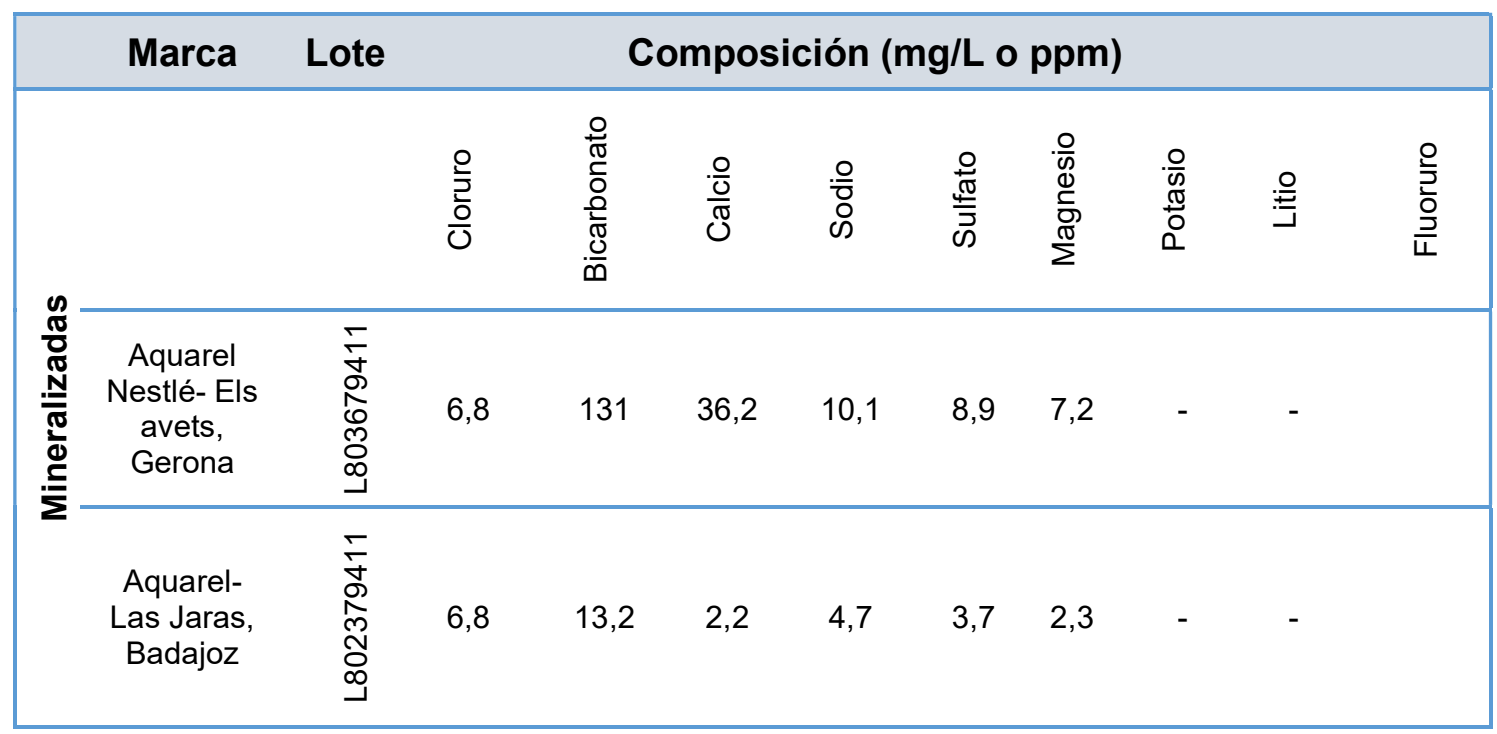




\begin{tabular}{|c|c|c|c|c|c|c|c|c|c|c|c|}
\hline & $\begin{array}{l}\text { Cortes de } \\
\text { Arenoso } \\
\text { con gas- } \\
\text { Alicante }\end{array}$ & $\begin{array}{l}\stackrel{\infty}{\infty} \\
\stackrel{0}{0} \\
0\end{array}$ & - & 267,1 & 86,8 & 7,7 & 16,0 & 7,8 & $<1$ & - & $<0,01$ \\
\hline & $\begin{array}{l}\text { Fuente } \\
\text { Liviana- } \\
\text { Cuenca }\end{array}$ & $\begin{array}{l}\text { ô } \\
\text { ष् } \\
\text { త్ }\end{array}$ & - & 273 & 67,4 & 0,8 & - & 18,5 & - & - & \\
\hline & $\begin{array}{c}\text { Fuente } \\
\text { Primavera - } \\
\text { Valencia }\end{array}$ & $\begin{array}{l}\infty \\
\stackrel{\infty}{\infty} \\
0 \\
\stackrel{D}{N} \\
\end{array}$ & 37 & 303 & 86 & 20 & - & 23 & - & - & \\
\hline & $\begin{array}{l}\text { Lanjarón - } \\
\text { Granada }\end{array}$ & 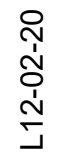 & - & 131 & 26,6 & 5,5 & - & 12,9 & - & - & \\
\hline & $\begin{array}{l}\text { Solán de } \\
\text { Cabras - } \\
\text { Cuenca }\end{array}$ & 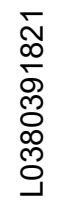 & - & 284 & 60,0 & 4,8 & - & 26,7 & 1,0 & - & \\
\hline & $\begin{array}{l}\text { Vichy } \\
\text { Catalán- } \\
\text { Gerona }\end{array}$ & 3 & 584,0 & 2081,0 & - & 1097,0 & 49,6 & - & 50,7 & 1,3 & \\
\hline$\frac{\mathscr{d}}{\frac{\pi}{0}}$ & $\begin{array}{l}\text { Neval- } \\
\text { Moratalla, } \\
\text { Murcia }\end{array}$ & 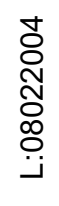 & 147 & 243 & 56,9 & 91,8 & 122 & 44,3 & - & - & \\
\hline$\Sigma$ & $\begin{array}{l}\text { Mondariz- } \\
\text { Pontevedra }\end{array}$ & 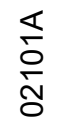 & 14,7 & - & 6,6 & 47,7 & - & 5,2 & - & - & 0,49 \\
\hline
\end{tabular}

\section{Aguas de abastecimiento}

Se recogieron $50 \mathrm{ml}$ de agua de consumo de 10 sitios diferentes de la Región de Murcia: Alcantarilla, Barrio La Merced (Murcia centro), Barrio Vista Alegre (Murcia centro), Cartagena, Churra (Murcia), El Palmar (Murcia), Espinardo (Murcia), Urb. La Alcayna (Molina de Segura), Llano de Brujas (Murcia) y Lorca. Las aguas se mantuvieron almacenadas en tubos de polietileno con tapón de rosca a temperatura ambiente, resguardadas de la luz del sol y de la humedad hasta el momento del análisis.

Como control se usó agua ultrapura obtenida con el Milli-Q® IQ 7000 (Merck KGaA, Darmstadt, Germany). Todas las muestras fueron recogidas y etiquetadas por un investigador que no intervino en las determinaciones, para hacer el estudio a ciego.

\section{Determinación de la concentración de fluoruro}

La determinación de flúor fue realizada con un electrodo ion-específico (Orion 9609 BNWP, Thermo Fisher Scientific Inc., Waltham, EE.UU.), conectado a un analizador de iones (Orion EA-940, Thermo Fisher Scientific Inc. Waltham, EE.UU.). El electrodo 
fue previamente calibrado con soluciones estándar desde 0,10 a $1,0 \mathrm{ppm} F$, mezclando $1 \mathrm{ml}$ de cada solución estándar con 1 mililitro de TISAB II (Hanna Instruments, Woonsocket, Rhode Island, EE.UU.) [1.0 M acetate buffer pH 5.0, 1.0 M $\mathrm{NaCl}$ and $0.4 \% \mathrm{CDTA}$. Las muestras de agua fueron tamponadas con una solución de TISAB II $(1: 1, \mathrm{v} / \mathrm{v})$. Antes de cada una de las lecturas, las muestras fueron agitadas con una vibradora (Classic Vortex Mixer, Velp Scientifica, Italia). Los resultados la lectura se recogieron en milivoltios $(\mathrm{mV})$ y se convirtieron a $\mathrm{mgF} / \mathrm{L}(\mathrm{ppm} \mathrm{F})$ gracias a la curva de calibración.

\section{RESULTADOS}

Las concentraciones de fluoruro en las aguas embotelladas variaron entre 0.04 a 0.50 ppm (Tabla 2). Salvo el agua de Vichy Catalán, el resto de aguas mineralizadas presentaron menor cantidad de fluoruro que las aguas minerales naturales.

Tabla 2. Concentración de fluoruro en aguas embotelladas minerales y mineralizadas consumidas en la Región de Murcia (mg/L o ppm).

\begin{tabular}{|c|c|c|}
\hline & Marca & $\mathbf{F}$ \\
\hline \multirow{8}{*}{ 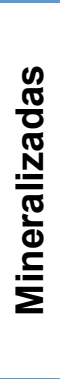 } & Aquarel Nestlé (Els avets, Gerona) & 0,10 \\
\hline & Aquarel (Las Jaras, Badajoz) & 0,04 \\
\hline & Cortes de Arenoso con gas (Alicante) & 0,08 \\
\hline & Fuente Liviana (Cuenca) & 0,1 \\
\hline & Fuente Primavera (Valencia) & 0,15 \\
\hline & Lanjarón (Granada) & 0,16 \\
\hline & Solán de Cabras (Cuenca) & 0,10 \\
\hline & Vichy Catalán (Gerona) & 0,50 \\
\hline \multirow{2}{*}{$\begin{array}{l}\frac{\mathscr{d}}{\mathbb{N}} \\
\frac{\mathbf{T}}{\mathbf{d}} \\
\frac{\Sigma}{\Sigma}\end{array}$} & Neval (Moratalla, Murcia) & 0,21 \\
\hline & Mondariz (Pontevedra) & 0,43 \\
\hline
\end{tabular}

De todas las aguas embotelladas solamente el agua mineral Cortés del Arenoso con gas de Alicante y la Mondariz de Pontevedra indicaron en su etiquetado la concentración de fluoruro.

En el agua de Mondariz, que declaraba contener $0.49 \mathrm{ppm}$, nosotros encontramos $0.43 \mathrm{ppm}$; y en el caso de la de Cortes del Arenoso, se hallaron $0.08 \mathrm{ppm}$ y en su etiquetado indicaba $<0.1$. El resto de las marcas de aguas embotelladas no indicaban la presencia de fluoruro y sí lo contenían.

Las aguas de abastecimiento de la región de Murcia han presentado valores de fluoruro variables, desde $<0.04 \mathrm{ppm}$, encontrado en el agua de Cartagena, hasta 0.28 ppm en el Barrio de Vista Alegre (Murcia centro) o en la Urbanización La Alcayna en Molina de Segura (Tabla 3). 
Tabla 3. Concentración de fluoruro en las aguas de abastecimiento de la Región de Murcia (mg/L o ppm).

\begin{tabular}{|lc|}
\hline Procedencia de las aguas de abastecimiento & Fluoruro \\
\hline Alcantarilla & 0,17 \\
\hline Barrio La Merced (Murcia centro) & 0,26 \\
\hline Barrio Vista Alegre (Murcia centro) & 0,28 \\
\hline Cartagena & $<0,04$ \\
\hline Churra (Murcia) & 0,18 \\
\hline El Palmar (Murcia) & 0,10 \\
\hline Espinardo (Murcia) & 0,09 \\
\hline Llano de Brujas (Murcia) & 0,23 \\
\hline Lorca & 0,18 \\
\hline Urb. La Alcayna (Molina de Segura) & 0,28 \\
\hline Agua ultrapura (control) & 0,01 \\
\hline
\end{tabular}

\section{DISCUSIÓN}

El flúor interfiere en los procesos de desmineralización y remineralización del diente, previniendo la caries. Para que pueda disminuir la pérdida de minerales debe estar presente en la cavidad oral en concentraciones bajas y constantes ${ }^{(12)}$. El agua es una de las principales fuentes de ingesta de flúor. Para los profesionales de la odontología, es fundamental conocer este aporte para poder prescribir con rigor los suplementos de fluoruro necesarios para una correcta prevención de la caries, sobre todo en niños en crecimiento.

Los resultados de nuestro estudio establecen que la concentración de fluoruro contenido en las aguas que se consumen en Murcia, tanto las embotelladas como las de abastecimiento, están por debajo de 0,6 ppm, concentración que la AAPD considera beneficiosa para la prevención de la caries sin riesgo de fluorosis ${ }^{(9)}$.

En 2014, el agua de la red pública en el 95\% de las ciudades españolas contenía una concentración de flúor inferior a 0,7 ppm ${ }^{(8)}$. En la Región de Murcia se dejó de añadir flúor al agua de abastecimiento en el año 2013, debido al uso generalizado de productos dentales que contienen flúor y al consumo de aguas embotelladas por parte del $50 \%$ de la población. Esta población podría conocer la cantidad de fluoruro que consume al día, pero el 50\% restante no tiene información sobre de la cantidad de flúor que consume ${ }^{(11)}$ ya que desconoce la cantidad de fluoruro que contiene el agua de abastecimiento. A pesar de que el agua de abastecimiento no está fluorada, hemos detectado una presencia de flúor por encima de los $0.2 \mathrm{ppm}$ en 4 localizaciones: los dos barrios de Murcia centro, la urbanización la Alcayna y Llano de Brujas. Aunque entre La Alcayna y el Barrio de Vista Alegre hay una separación de $9.1 \mathrm{Km}$, la concentración de fluoruro ha sido la misma, 0.28 ppm.

La normativa en España no obliga a que las aguas minerales y mineralizadas indiquen en su etiquetado la cantidad de flúor que contienen cuando su concentración es inferior a $1.5 \mathrm{mg} / \mathrm{L}$. Sin embargo, cuando la concentración es superior a $1.5 \mathrm{mg} / \mathrm{L}$, deberían incluir en su etiquetado, inmediatamente al lado de la denominación de venta y en caracteres claramente visibles, la indicación «contiene más de $1.5 \mathrm{mg} / \mathrm{L}$ de flúor: no adecuada para el consumo regular de los lactantes y niños menores de siete años» (RD 140/2003 de 7 de febrero) ${ }^{(13)}$. Y según el Real Decreto 1798/2010 de 30 
de diciembre (10), se considera que el agua con más de 1 ppm puede llamarse "fluorada o que contiene flúor", no siendo obligatorio especificar la concentración de flúor en la etiqueta. Sin embargo, la Organización de las Naciones Unidas para la Alimentación y la Agricultura (FAO) establece que, en caso de que el producto contenga más de $1 \mathrm{mg} / \mathrm{L}$ de fluoruro, en la etiqueta debería figurar, como parte del nombre del producto, o muy cerca de éste, o en lugar visible, las palabras "contiene fluoruro" (14).

Las empresas que comercializan el agua embotellada, deberían especificar su contenido en flúor y permitir que el consumidor tome la decisión de compra en base a una información verídica con respecto al consumo de agua potable fluorada versus "no fluorada" (15-17). Sólo en dos de las aguas embotelladas analizadas en nuestro estudio aparece reflejado en el etiquetado la presencia de flúor. En las de Cortés de Arenoso con gas (Alicante) se indica $<0.1 \mathrm{ppm}$ de fluoruro, coincidiendo con la concentración que nosotros hemos detectado (0.08 ppm de flúor) y el agua de Mondariz (Pontevedra) 0.49 ppm y en nuestro estudio se detectaron 0.43 ppm.

Todas las aguas embotelladas analizadas presentaron un contenido de fluoruro seguro para el consumo y sin riesgos de aparición de fluorosis dental, ya que la máxima concentración de fluoruro encontrada fue del $0.50 \mathrm{ppm}$ en el agua mineralizada Vichy Catalán (Gerona). Sin embargo, estas concentraciones encontradas tampoco son útiles para prevenir la caries. El Ministerio de Sanidad establece que el contenido de flúor en el agua, para que presente un efecto protector contra la caries dental, debe oscilar entre 0.6 y $0.8 \mathrm{ppm}$ para las ciudades con temperaturas entre $26.4{ }^{\circ} \mathrm{C}$ y $32.5^{\circ} \mathrm{C}$, como Murcia (10). Debemos tener en cuenta la necesidad de los suplementos de flúor para aquellos niños que beban agua con menos de 0,6 ppm $\mathrm{F}$ y que presenten riesgo alto de caries ${ }^{(9)}$. Pero antes de prescribir suplementos de flúor hay que analizar el consumo diario total de flúor del niño que puede tener diferentes fuentes, como el agua (en casa, en el colegio, de abastecimiento y embotellada), los alimentos y otras bebidas, la fórmula de leche infantil, la pasta de dientes, etc.

\section{CONCLUSIONES}

El agua de consumo, tanto de abastecimiento como embotellada, en la Región de Murcia contiene fluoruro en cantidades insuficientes para la prevención de caries. No obstante, habría que tenerlas en cuenta a la hora de prescribir los suplementos de flúor en niños.

\section{REFERENCIAS}

1. Corrêa-Faria P, Paixão-Gonçalves S, Paiva SM, Pordeus IA. Incidence of dental caries in primary dentition and risk factors: a longitudinal study. Braz Oral Res. 2016;30(1). Disponible en: http://dx.doi.org/10.1590/1807-3107BOR2016.vol30.0059

2. Alkhtib A, Ghanim A, Temple-Smith M, Messer LB, Pirotta M, Morgan M. Prevalence of early childhood caries and enamel defects in four and five-year old Qatari preschool children. BMC Oral Health. 2016;16(1):73. Disponible en: http://dx.doi.org/10.1186/s12903-016-0267-z 
3. Kassebaum NJ, Smith AGC, Bernabé E, et al. Global, Regional, and National Prevalence, Incidence, and Disability-Adjusted Life Years for Oral Conditions for 195 Countries, 1990-2015: A Systematic Analysis for the Global Burden of Diseases, Injuries, and Risk Factors. J Dent Res. 2017;96(4):380-387. Disponible en: http://dx.doi.org/10.1177/0022034517693566

4. Bravo Pérez M, Almerich Silla JM, Ausina Márquez V, Avilés Gutiérrez P, Blanco González JM, Canorea Díaz E., et al. Encuesta de Salud Oral en España 2015. Published online June 1, 2016. Accessed May 8, 2020. Disponible en: http://diposit.ub.edu/dspace/handle/2445/103211

5. Pepelascov DE, Fujimaki M, Cury JA, Tabchoury CPM, Villalobos JUG, Terada RSS. Fluoride concentration and labeling requirements of mineral bottled water from Brazil. Vigilância Sanitária Em Debate Soc Ciênc Tecnol. 2017;5(3):24-29. Disponible en: http://dx.doi.org/10.22239/2317-269x.00871

6. Burt BA. The changing patterns of systemic fluoride intake. $J$ Dent Res. 1992;71(5):1228-1237.

Disponible

en: http://dx.doi.org/10.1177/00220345920710051601

7. Ramires I, Grec RH da C, Cattan L, Moura PG de, Lauris JRP, Buzalaf MAR. Evaluation of the fluoride concentration and consumption of mineral water. Rev Saude Publica. 2004;38(3):459-465. Disponible en: http://dx.doi.org/101590/s0034-89102004000300018

8. Maraver F, Vitoria I, Almerich-Silla JM, Armijo F. Fluoride content of bottled natural mineral waters in Spain and prevention of dental caries. Aten Primaria. 2015;47(1):15-24. Disponible en: http://dx.doi.org/10.1016/j.aprim.2014.04.003

9. BP_FluorideTherapy.pdf. Accessed $\quad$ May 2020. https://www.aapd.org/media/Policies_Guidelines/BP_FluorideTherapy.pdf

10. Real Decreto $1798 / 2010$, de 30 de diciembre, por el que se regula la explotación y comercialización de aguas minerales naturales y aguas de manantial envasadas para consumo humano. :27. Gobierno de España: Ministerio de la Presidencia, relaciones con las cortes e igualdad. Disponible en:https://www.boe.es/buscar/act.php?id=BOE-A-2011-971; 2011 [consultado 21.03.2020].

11. Marín Camaches MD, Pacheco Martínez F, Martínez Gambín R, Gómez Campoy E, Gutierrez Molina C. [Water fluoridation in the Region of Murcia]. Rev Esp Salud Publica. $\quad 1998 ; 72(2): 91-101$.

Disponible

en: https://www.ncbi.nlm.nih.gov/pubmed/9643064

12. Kanduti D, Sterbenk $P$, Artnik B. Fluoride: a review of use and effects on health. Mater Socio-Medica. 2016;28(2):133-137. Disponible en: http://dx.doi.org/10.5455/msm.2016.28.133-137

13. Real Decreto 140/2003, de 7 de febrero, por el que se establecen los criterios sanitarios de la calidad del agua de consumo humano. :41. Gobierno de España: Ministerio de la Presidencia, relaciones con las cortes e igualdad; Disponible en: https://www.boe.es/buscar/act.php?id=BOE-A-2003-3596; 2003 [consultado 21.03.2020].

14. FAO/OMS. Programa conjunto FAO/OMS sobre normas alimentarias. Comisión del Codex Alimentarius [sede Web]. Roma: FAO/OMS; 1999. CX 5/40. Disponible en: $\quad$ http://www.fao.org/tempref/codex/Reports/Alinorm06/al2903As.pdf. [consultado 12.04.2020]

15. Lalumandier JA, Ayers LW. Fluoride and bacterial content of bottled water vs tap water. Arch Fam Med. 2000;9(3):246-250. Disponible en: http://dx.doi.org/10.1001/archfami.9.3.246 
16. Cochrane NJ, Saranathan S, Morgan MV, Dashper SG. Fluoride content of still bottled water in Australia. Aust Dent J. 2006;51(3):242-244. Disponible en: http://dx.doi.org/10.1111/j.1834-7819.2006.tb00436.x

17. Mythri H, Chandu GN, Prashant GM, Subba Reddy VV. Fluoride and bacterial content of bottled drinking water versus municipal tap water. Indian J Dent. 2010;21(4):515-517. Disponible en: http://dx.doi.org/10.4103/0970-9290.74223 